\title{
Long-term follow-up of surgical resection alone for primary intracranial rostrotentorial tumors in dogs: 29 cases (2002-2013)
}

\author{
Anna Suñol ${ }^{1}$, Joan Mascort ${ }^{1}$, Cristina Font ${ }^{1,2}$, Alicia Rami Bastante ${ }^{3}$, Martí Pumarola ${ }^{4}$ \\ and Alejandro Lujan Feliu-Pascual ${ }^{1,5, *}$ \\ ${ }^{1}$ Hospital Ars Veterinaria, carrer Cavallers n ${ }^{\circ} 37,08034$, Barcelona, Spain \\ ${ }^{2}$ Hospital Canis Girona. Carrer Can Pau Birol, 38. 17006 Girona, Spain \\ ${ }^{3}$ Neuroscience Institute of Barcelona. Universitat Autònoma de Barcelona, edifici M1, campus de Bellaterra E- \\ 98193, Cerdanyola del Vallès, Spain \\ ${ }^{4}$ Department of Animal Medicine and Surgery, Veterinary Faculty, Universitat Autonoma de Barcelona, 08193 \\ Bellaterra (Cerdanyola del Vallès), Barcelona, Spain \\ ${ }^{5}$ AÚNA Especialidades Veterinarias, Calle Algepser 22-1, 46980 Paterna, Valencia, Spain
}

\begin{abstract}
Intracranial neoplasia is frequently encountered in dogs. After a presumptive diagnosis of intracranial neoplasia is established based on history, clinical signs and advanced imaging characteristics, the decision to treat and which treatment to choose must be considered. The objective of this study is to report survival times (ST) for dogs with intracranial meningiomas and gliomas treated with surgical resection alone (SRA), to identify potential prognostic factors affecting survival, and to compare the results with the available literature. Medical records of 29 dogs with histopathologic confirmation of intracranial meningiomas and gliomas treated with SRA were retrospectively reviewed. For each dog, signalment, clinical signs, imaging findings, type of surgery, treatment, histological evaluation, and ST were obtained. Twenty-nine dogs with a histological diagnosis who survived $>7$ days after surgery were included. There were 15 (52\%) meningiomas and 14 (48\%) gliomas. All tumors had a rostrotentorial location. At the time of the statistical analysis, only two dogs were alive. Median ST for meningiomas was 422 days (mean, 731 days; range, 10-2735 days). Median ST for gliomas was 66 days (mean, 117 days; range, 10-730 days). KaplanMeier analysis indicated that ST was significantly longer for meningiomas than for gliomas $(P<0.05)$. A negative correlation between the presence of a midline shift and ST $(P=0.037)$ and ventricular compression and ST $(P=0.038)$ was observed for meningiomas. For gliomas, there were no significant associations between ST and any of the variables evaluated. In conclusion, the results of this study suggest that, for dogs that survived $>7$ days postoperatively, SRA might be an appropriate treatment, particularly for meningiomas, when radiation therapy is not readily available. Also, the presence of midline shift and ventricular compression might be negative prognostic factors for dogs with meningiomas.
\end{abstract}

Keywords: Dog, Glioma, Intracranial tumor, Meningioma, Survival time.

\section{Introduction}

Intracranial neoplasia is frequently encountered in dogs (Song et al., 2013) and represent a major cause of morbidity and mortality (Dickinson, 2014). Meningiomas are the most commonly reported primary intracranial neoplasm in this species, followed by glial tumors, including astrocytomas, oligodendrogliomas, and oligoastrocytomas (Snyder et al., 2006; Sessums and Mariani, 2009; Song et al., 2013). After a presumptive diagnosis of intracranial neoplasia is established based on history, clinical signs and advanced imaging characteristics, the decision to treat and which treatment to choose must be considered. The therapeutic modalities most commonly available for veterinary patients with intracranial neoplasia include palliative care, surgery, chemotherapy and radiation therapy, all of which can be used either alone or in combination (Dickinson, 2014). Definitive diagnosis of intracranial tumors is based on histopathologic examination, (Dickinson, 2014) typically following the World Health Organization (WHO) classification system published in 2007 (Louis et al., 2007).

Palliative care for intracranial tumors often involves glucocorticoids targeting peritumoral edema together with antiepileptic drugs to control seizures, (Dickinson, 2014) which is the most common presenting complaint for intracranial tumors in dogs (Bagley et al., 1999; Snyder et al., 2006).

Mean survival time (ST) reported for dogs with rostrotentorial meningiomas that received only palliative treatment ranged from 54 to 195 days, (Turrel et al., 1984; Foster et al., 1988; Bilderback et al., 2006)

*Corresponding Author: Alejandro Lujan Feliu-Pascual. Hospital Ars Veterinaria, Carrer Cardedeu n³, 08023, Barcelona, 
whereas a median ST of 45 days was reported for gliomas (Adams et al., 2005).

Efficacy of surgical cytoreduction of intracranial tumors is highly operator and equipment dependent (Dickinson, 2014). Most published information is related to more easily accessible canine and feline meningiomas, with only anecdotal data for other tumor types such as gliomas (Dickinson, 2014). Surgical excision alone for canine meningiomas resulted in median ST of 180-210 days (Kostolich and Dulisch, 1987; Niebauer and Dayrell-Hart, 1991; Axlund et al., 2002; Bilderback et al., 2006; Rossmeisl, 2014). Neurosurgical treatment using devices such as intracranial endoscopy or ultrasonic surgical aspirator, which either improve intraoperative visualization or facilitate tumor excision, have been associated with longer median ST after surgical resection for intracranial meningiomas, with ST ranging from 1260 to 2100 days. (Greco et al., 2006; Klopp and Rao, 2009; Rossmeisl, 2014).

Currently, no meaningful conclusions can be made from published data relating to surgery of intraaxial tumors (Dickinson, 2014) and, to the best of the authors' knowledge, a retrospective or prospective paper looking for ST for gliomas treated with surgical resection alone (SRA) has not been previously reported.

There is little information available about the efficacy of chemotherapeutic agents for canine intracranial neoplasia. Most data relate to the use of nitrosureabased alkylating agents, such as lomustine (CCNU) and carmustine, or the ribunucleotide reductase inhibitor hydroxyurea (Dickinson, 2014). Anecdotal published data show apparent survival benefits and occasional responses with survival of many months in some cases (Jung et al., 2006; Tamura et al., 2007).

However, a large retrospective study suggested no benefit from lomustine chemotherapy compared to palliative care (93 days versus 60 days) for brain masses diagnosed via computed tomography and without a histologic diagnosis (Van Meervenne and Verhoeven, 2014).

Radiation therapy has become a mainstay of treatment for intracranial neoplasia in both human and veterinary patients, either as a primary or adjunctive treatment (Dickinson, 2014). Radiotherapy has demonstrated to improve ST in presumptive and confirmed meningiomas, with ST ranging from 240 to 577 days (Turrel et al., 1984; Evans et al., 1993; Axlund et al., 2002; Bley et al., 2005; Griffin et al., 2014; Rossmeisl, 2014). Radiation therapy has been reported to be beneficial when compared to SRA for the treatment of meningiomas (Axlund et al., 2002).

A study including 12 dogs with gliomas treated with radiotherapy only, showed a median ST of 255 days (Adams et al., 2005). In this same study, the combination of surgery and radiotherapy increased the median ST to 300 days (Adams et al., 2005). A large prospective study analyzing the outcome, ST, and complications of the different treatment modalities in veterinary medicine is lacking. Consequently, the information necessary to choose a particular treatment is mainly based on published clinical studies with a limited number of cases with variable treatment modalities and follow-up, and the personal experience of the neurologist/neurosurgeon (Dickinson, 2014).

Until a large prospective study is designed, conducted and published, our aim was to report the long-term survival in a cohort of dogs with primary brain tumors (meningiomas and gliomas), where standard surgical resection was the main treatment modality due to the limited availability of radiotherapy, and to compare our results with previously published studies.

We also aimed to assess potential prognostic factors pertaining to signalment, magnetic resonance imaging (MRI) characteristics or type of tumor associated with ST. We hypothesized that ST for meningiomas treated with SRA would be longer than previously reported and that ST for gliomas following SRA would be longer than the ST reported with palliative care across previous publications.

We also hypothesized that the tumor type and some specific MRI characteristics, such as the presence of a midline shift, peritumoral edema and ventricular compression, would have a negative influence on ST.

\section{Case selection criteria}

\section{Materials and Methods}

Medical records of dogs examined at our hospital from April 2002 to December 2013 were retrospectively reviewed. All dogs with a histopathologic diagnosis of primary intracranial tumor following surgical resection were included.

\section{Medical records review}

Information obtained from the medical records included signalment, type and duration of clinical signs, tumor location, MRI findings, type of surgery, duration of hospitalization, WHO histopathological classification, date of recurrence of clinical signs, and date and cause of death. If not available in the medical records, referring veterinarians and clients were contacted by telephone in order to obtain follow-up information.

\section{Procedures and follow-up}

For each dog, hematology, serum biochemistry profile, and systemic tumor staging (including 3-view thoracic radiographs and abdominal ultrasound) were performed to rule out potential underlying or concurrent diseases in preparation for surgery. Additional ancillary testing was performed as indicated.

For all dogs, a tentative diagnosis of intracranial neoplasia was obtained following $0.25 \mathrm{~T}$ MRI of the 
brain (Esaote VetMR Grande, Genoa, Italy) and cerebrospinal fluid analysis, both performed under general anesthesia.

A second year neurology resident and a board-certified neurologist examined all MR images. For each MRI study, tumor type (suspected meningioma versus glioma), neuroanatomical location (frontal, parietal, temporal), and size of the presumptive brain tumor, as well as any secondary changes observed, including the presence of a midline shift, peritumoral edema and compression of the ventricular system, were recorded. The percentage of midline shift was calculated on transverse T2-weighted (T2W) fast spin echo images. The midline shift was measured as the distance of maximum shift from the midline using commercial software (Osirix version 4.1.2 DICOM viewer, Pixmeo SARL, Bernex, Switzerland) as previously reported (Beltran et al., 2014).

This value was multiplied by 100 and divided by the distance from the midline to the convexity surface at the same level to calculate the percentage of midline shift (Beltran et al., 2014). Peritumoral edema was classified as present or absent based on T2W images. If present, it was graded as peritumoral if it extended $\leq 10$ $\mathrm{mm}$ beyond the tumor margin on $\mathrm{T} 2 \mathrm{~W}$ images or as diffuse if it extended for $>10 \mathrm{~mm}$ beyond the tumor margin (Sturges et al., 2008).

Ventricular system compression was evaluated in dorsal T1 post contrast images at the level of corpus callosum. If there were any abnormality in the symmetry, location or size of the ventricles that was attributed to direct tumor compression or surrounding edema, it was classified as present. If there were no abnormality it was classified as absent (Fig. 1).

Animals were anesthetized for surgery using standard hospital protocols. Preanesthetic medications included a combination of methadone $(0.2-0.4 \mathrm{mg} / \mathrm{kg}$ SC/IM, Metasedin, Laboratorio Esteve) and acepromazine (0.05-0.1mg/kg SC/IM, Equipromacina, Fatro Iberica SA). Anesthesia was induced with thiopental (3.0-6.6 $\mathrm{mg} / \mathrm{kg}$, IV, Tiobarbital, Braun) or propofol (4.0-6.0 $\mathrm{mg} / \mathrm{kg}$, IV, Propofol Hospira, Hospira SL) and diazepam (0.5 mg/kg IV, Valium, Roche Farma SA) and maintained with isoflurane and oxygen (1-2\% Isoflo, Laboratorio Esteve). All animals received perioperative antibiotics (cefazolin, $25 \mathrm{mg} / \mathrm{kg}$, IV, every $90 \mathrm{~min}$, Cefazolin Normon, Laboratorios Normon SA).

Two board-certified neurologists performed all surgical resections using magnifying loupes and a non-vibrating surgical aspirator (Ferguson suction tube, Instrumevet, Barcelona, Spain). Surgical approaches and operative techniques were performed according to the nature and location of the lesion in order to remove the maximal volume of tumor observed macroscopically.

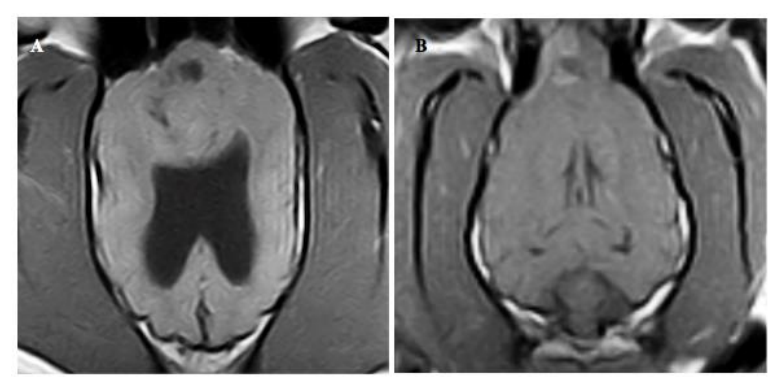

Fig. 1. T1W post contrast dorsal images at the level of corpus callosum. (A): Ventricular compression considered as present. (B): Ventricular compression considered as absent.

Once the tumor was located, surgical resection was performed as follows: for meningiomas, once a clear dissection plane was identified, blunt separation and removal of the macroscopic neoplastic tissue was achieved with nerve hooks, flat curettes, and bipolar cautery.

For gliomas, since no clear dissection plane was evident, a biopsy was obtained using curettes and the remaining tumor was removed using a non-vibrating surgical aspirator (Ferguson suction tube, Instrumevet, Barcelona, Spain) until removal was considered macroscopically complete. Hemorrhage was controlled via bipolar electrocautery and application of hemostatic sponges, when appropriate. Intraoperative mannitol was administered prior to dural opening when advanced imaging suggested increased intracranial pressure. The dura mater was not sutured but hemostatic sponges were placed tightly over the craniectomy defect. The bone flap was only replaced in $\operatorname{dog}$ s that underwent transfrontal craniotomy. Soft tissues were closed routinely.

While in recovery, dogs were positioned with the head above the level of the heart until alert and responsive, and were closely monitored for seizure activity, decreased mentation, or hypoventilation.

For the histopathological study, a biopsy sample was obtained during the surgical procedure and fixed in $10 \%$ formalin. A board-certified pathologist evaluated all tumors.

Animals were recovered in the intensive care unit for administration of supportive care, broad-spectrum antibiotics, and opioid analgesia (methadone at 0.2-0.4 $\mathrm{mg} / \mathrm{kg} \mathrm{SC}$ (Metasedin, Laboratorio Esteve), or fentanyl continuous rate infusion at 3-6 $\mu \mathrm{g} / \mathrm{kg} / \mathrm{h}$ IV (Fentanest, Kern pharma) until discharge. After surgery, treatment included oral anticonvulsants and glucocorticoids for epileptic seizure control and peritumoral edema reduction, respectively. Regular monitoring of anticonvulsant blood level was conducted as indicated. Median hospital stay was recorded and the first postoperative control was performed at our hospital within the first week after surgery. 
For ST analysis, recurrence of clinical signs and date and cause of death were obtained from the medical records, or from the referring veterinarian or client via telephone conversation.

\section{Statistical analyses}

For the purpose of the statistical analyses, dogs were classified into two groups depending on the histopathological results. Group I included all dogs diagnosed with meningiomas and group II included all dogs with gliomas.

Survival time was defined as the time from surgery until the date of death. Dogs that died within 7 days of surgery from complications directly related to the surgical procedure and those with incomplete medical records were excluded from the survival analysis. Dogs that died of causes unrelated to tumor progression were censored. For dogs that were still alive at the time of data analysis, the corresponding number of days between surgery and time where data was collected was considered.

The Kaplan-Meier product limit method was used to calculate median ST by means of a commercially available statistical analysis software (Statistical Package for the Social Sciences v.20 (SPSS Inc., Chicago, IL)(SPSS)). The effect of age, sex, and type of tumor (meningioma versus glioma) on ST was evaluated.

Categorical data was evaluated using Fisher's exact test. Shapiro-Wilk test confirmed the majority of the continuous variables were not normally distributed; therefore, a non-parametric test (Kruskal-Wallis) was used. In addition, for each MRI study the effects of the severity of the midline shift, perilesional edema, and ventricular compression on the ST were evaluated. Severity of the midline shift and its potential correlation with ST was evaluated for each group separately by means of Pearson's correlations. An ANOVA test was used to evaluate the association between perilesional edema and ST, and the effect of ventricular compression on ST was evaluated using a $t$-test. Significance level was set at $P$ value $\leq 0.05$.

\section{Results}

During the study period, SRA was performed on 35 dogs with intracranial tumors. Four dogs (3 with a meningioma and 1 with a glioma) were excluded from the study because they died within 7 days following surgical resection. Two of them died secondary to middle cerebral artery thrombosis occurring 4 and 12 hours after surgery. Post-mortem examination was performed in both cases. The other 2 dogs were euthanized due to postoperative aspiration pneumonia, and intraoperative hypotension and cardiorespiratory failure, respectively. Two additional dogs were excluded because of incomplete medical records. Therefore, 29 dogs with a histopathologically confirmed primary intracranial neoplasia survived $>7$ days following surgical resection and were included in the study. There were $15(52 \%)$ meningiomas (group I) and $14(48 \%)$ gliomas (group II).

In group I, $6(40 \%)$ dogs were male (4 intact, 2 neutered) and $9(60 \%)$ were female (3 intact, 6 spayed). Median age at initial presentation was 11 years (mean, 10.3 years; range, 5-14.6 years). Seven dogs were purebreds and 8 were mixed breeds. Only German shepherd dogs were represented by $>1 \operatorname{dog}(n=2)$. In Group II, 8 (57\%) dogs were male (5 intact, 3 neutered) and $6(43 \%)$ were female (1 intact, 5 spayed). Median age at initial presentation was 8.8 years (mean, 8.6 years; range, 6-12.5 years). Eight dogs in group II were purebreds and 6 were mixed breeds. Breeds represented by $>1$ dog included Golden Retriever $(n=4)$ and Boxer $(n=2)$. Clinical signs reflected the neuroanatomic location of the tumor in all dogs. The most common clinical signs were seizures and behavioral changes, present in 24 of 29 dogs. Contralateral motor and sensory deficits were noted in 3 of 29 dogs. Two dogs also showed clinical signs consistent with central vestibular disease including obtundation, head tilt, tetraparesis and spontaneous nystagmus. The duration of clinical signs prior to diagnosis varied widely, ranging from 1 day to 1 year (mean, 54 days).

Three types of surgical approaches were used to resect the 29 intracranial masses visualized on MRI including transfrontal (20 dogs), frontoparietal (5 dogs), and parietotemporal (4 dogs) approaches. Median hospital stay for all surviving dogs was 3 days (range, 2-6 days). All dogs but two had one surgery performed. Two dogs with meningiomas underwent more than one surgery. One of these two dogs was still alive 2735 days (7.5 years) after the first surgery, with a total of three surgeries performed. A second surgery was performed 1440 days after first one, and a third surgery 450 days after the second surgical procedure. This dog had a Grade I psammomatous meningioma, with little histopathological change between the first and second biopsies. The other $\mathrm{dog}$ had a histopathological confirmation of transitional meningioma, and died 1740 days (4.7 years) after the first surgery having had a second surgery performed 1224 days after the first tumor resection. In addition, two dogs were censored because they died from causes unrelated to tumor progression. One dog, which had a meningioma, died 768 days after surgery due to renal failure. One dog with an oligodendroglioma died 15 days after surgery after developing a pericardial effusion apparently unrelated to the tumor. Post-mortem examination was performed and no underlying etiology for the pericardial effusion was found.

A board-certified pathologist evaluated all tumors and classified them according to the WHO classification system (Louis et al., 2007). There were 15 (52\%) meningiomas and $14(48 \%)$ gliomas. 

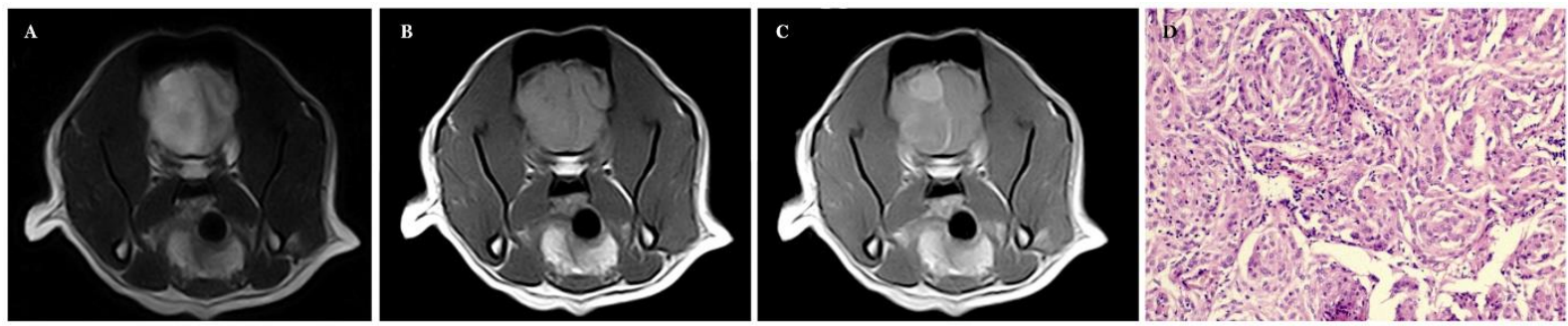

Fig. 2. Grade I canine frontal lobe transitional meningioma. Transverse $\mathrm{T} 2 \mathrm{~W}, \mathrm{~T} 1 \mathrm{~W}$ and $\mathrm{T} 1 \mathrm{~W}$ post contrast $(\mathrm{T} 1+\mathrm{C})$ magnetic resonance image at the level of frontal lobe. (A): T2W: The tumor shows hyperintensity respect the grey matter and peritumoral edema. (B): T1W: the tumor shows isointensity respect the grey matter. (C): $\mathrm{T} 1 \mathrm{~W}+\mathrm{C}$ : the tumor enhanced homogenously and there is also meningeal enhancement. Also noted the midline shift present in all three sequences. (D): Transverse T1WHematoxylin and eosin stain showing whorls of spindle-shaped cells. Original magnification 40x.
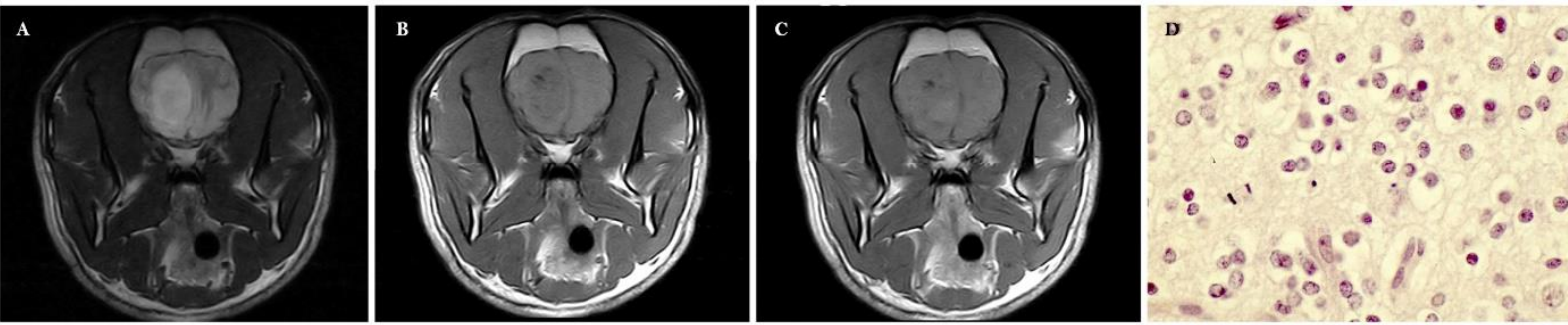

Fig. 3. Grade III canine frontal lobe oligodendroglioma. Transverse $\mathrm{T} 2 \mathrm{~W}$, $\mathrm{T} 1 \mathrm{~W}$ and $\mathrm{T} 1 \mathrm{~W}$ post contrast $(\mathrm{T} 1 \mathrm{~W}+\mathrm{C})$ magnetic resonance image at the level of the frontal lobes. (A): T2W: Well-defined T2-hyperintensity lesion respect the grey matter. (B): T1W: The lesion shows heterogeneously T1-hypointensity respect the grey matter. (C): T1W+C: Mild enhancement in the medial ventral part of the lesion. Also noted the midline shift present in all three sequences. (D): Hematoxylin and eosin stain showing the honeycomb pattern characteristic of these tumors. Original magnification 400x.

All tumors had a rostrotentorial location. Meningioma subtypes represented included transitional (7), psammomatous (1), fibroblastic (1), meningothelial (1), atypical (2), and anaplastic (3). According to the WHO classification system (Louis et al., 2007), there were 10 Grade I meningiomas, 2 Grade II meningiomas, and 3 Grade III meningiomas (Fig. 2). The gliomas resected included oligodendrogliomas (8), astrocytomas (4), and anaplastic gliomas (2). There was 1 Grade I glioma, 5 Grade II gliomas, and 8 Grade III gliomas (Fig. 3).

Median ST for dogs with meningiomas was 422 days (mean, 731 days; range, 10-2735 days). Median ST for dogs with gliomas was 66 days (mean, 117 days; range, 10-730 days). Kaplan-Meier survival analysis indicated that ST for dogs with meningiomas treated with SRA was statistically longer than ST for dogs with gliomas treated with SRA ( $\mathrm{F}=0.024 ; P<0.05)$ (Fig. 4).

In the meningioma group, $6 / 15(40 \%)$ had $\geq 20 \%$ of midline shift displacement, whereas 5/14 (36\%) gliomas showed this feature. A negative correlation between the presence of a midline shift and ST was observed for meningiomas $(P=0.037)$. In the meningioma group, $14 / 15$ (93\%) had $\leq 10 \%$ of edema, with only 1 dog meningioma having $>10 \%$ of edema. In the glioma group, $4 / 14(28.6 \%)$ had $>10 \%$ of edema, 9/14 (64.3\%) had <10\% of edema, and 1/14 (7.1\%) had no observable edema on MRI. No significant association between the presence of peritumoral edema and ST was observed for either type of tumor. Ten of $15(66.7 \%)$ meningiomas had ventricular compression in comparison with $9 / 14$ cases $(64.3 \%)$ in the glioma group. A statistically significant negative association between the presence of ventricular compression and ST was observed for meningiomas $(P=0.038)$. For gliomas, there were no significant associations between any of the MRI variables and ST. Among all data collected, only tumor histopathology (meningioma vs. glioma) (Fig. 3), and the presence of a midline shift and ventricular compression in meningiomas had a significant influence on ST.

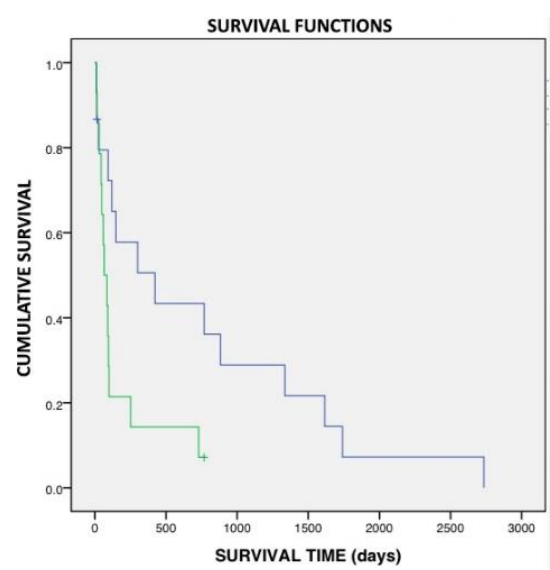

Fig. 4. Kaplan-Meier survival curve. Median survival times in dogs with intracranial primary tumors treated with SRA for meningiomas (blue) compared with gliomas (green). 


\section{Discussion}

The results of the present study suggest that in dogs with intracranial rostrotentorial meningiomas, SRA might result in ST longer than previously reported. In our study, dogs with meningiomas treated with SRA had a median ST of 422 days, which compares favorably to previously published median ST after standard surgical resection, which have typically ranged from 180 to 210 days (Kostolich et al., 1987; Niebauer et al., 1991; Axlund et al., 2002; Bilderback et al., 2006; Rossmeisl, 2014). In addition, we report ST for 14 gliomas treated with SRA, with a median survival of 66 days, which is slightly longer than previously reported with palliative treatment (Adams $e t$ al., 2005). Of the secondary changes evaluated on MRI, the presence of a midline shift and ventricular compression were significantly associated with a lower ST in dogs with meningiomas treated with SRA, suggesting a negative prognostic implication. For the glioma group, we did not observe any associations between MRI characteristics and ST.

All the tumors included in our study had a rostrotentorial location, which might have influenced the decision to operate as well as the long-term ST. In general, rostrontentorial tumors have been reported to have a better prognosis than infratentorial neoplasms (Dickinson, 2014). Focusing on meningiomas, the available literature has reported median ST of 180-210 days after SRA (Kostolich et al., 1987; Niebauer et al., 1991; Axlund et al., 2002; Bilderback et al., 2006; Rossmeisl, 2014). However, dogs with meningiomas treated with SRA in this study achieved a longer median ST (median, 422 days; mean, 731 days). Similar to previous studies, (Axlund et al., 2002; Klopp et al., 2009) dogs that died within 7 days after surgery were not included in the ST analyses. However, not all previously published studies followed the same criteria, making comparisons between studies challenging. Most of the reports available described surgical resection of olfactory bulb or frontal lobe meningiomas, similar to our study. One of the first reports describing intracranial surgery in dogs (Kostolich et al., 1987) included 4 olfactory meningiomas treated with surgery with a median ST of 135 days, with all dogs surviving beyond the first 7 days after surgery. The longest median ST described for dogs with meningiomas treated with SRA was 210 days, based on 18 cases (Axlund et al., 2002). This report excluded from the survival analyses those dogs that died within the first 7 days following surgical resection, similar to the method that was followed in the present study. The most recent report describing SRA for the treatment of canine brain tumors (Bilderback $e t$ al., 2006) reported a median ST of 201 days. However, this study comprised a small number of cases and only included 5 meningiomas. Moreover, one of these 5 dogs died during the third day after surgery. With the data available it was difficult to obtain the exact ST for the remaining 4 patients, although a median ST of 204 days was inferred from the data provided. Overall, our study achieved a longer median ST for dogs with meningiomas surviving $>7$ days after SRA, in comparison to previous studies that used similar criteria to calculate ST (Axlund et al., 2002). In our study the two cases with meningioma that had more than one surgery have had a slightly positive influence in our ST. If these animals had been included only for the first procedure, the median ST for meningiomas would also have been 422 days, with a mean of 611 days.

History and signalment from the dogs included in this study were similar to previous studies, including a majority of older dogs, consistent with the previously reported average age at presentation of 9.5-11 years (Bagley et al., 1999; Axlund et al., 2002; Song et al., 2013; Dickinson, 2014). Also, both genders appeared equally represented, which matches the lack of a sex predisposition described in the literature (Dickinson, 2014). It has been suggested that meningiomas are overrepresented in Golden Retrievers, Boxers, and Miniature Schnauzers, and that astrocytomas and oligodendrogliomas are overrepresented in brachycephalic breeds (Dickinson, 2014). In our study, we did not observe a clear breed predisposition in the meningioma group, but there were 4 gliomas diagnosed in Golden Retrievers and 3 in brachycephalic dogs (2 Boxers, 1 French Bulldog). Seizures were the most common initial complaint in our study with 24 of 29 dogs initially examined for this reason. This is also consistent with previous publications (Bagley et al., 1999; Snyder et al., 2006) and it is not surprising considering the rostrontentorial origin of all tumors included in the present study. Of the signalment variables analyzed in this study (age, sex, and type of tumor), only tumor type was found to have a significant influence on ST, with meningiomas having longer ST than gliomas.

In humans, meningiomas are histologically categorized as Grade I, II, and III according to the 2007 WHO classification (Louis et al., 2007). Grade I meningiomas include psammomatous, transitional, meningothelial and fibroblastic subtypes. Grade II meningiomas include atypical and choroid subtypes. Grade III meningiomas include anaplastic and papillary subtypes and are considered to be the most aggressive (Louis et al., 2007). WHO classification of meningiomas in domestic animals does not include categorization of subtypes into different grades (Koestner et al., 1999) therefore, the use of the human classification has been suggested (Sturges et al., 2008; Mandara et al., 2009). Due to the limited sample size of our study, we were unable to evaluate any associations between meningiomas subtypes/grades and survival. 
However, most of the meningiomas included in this study were classified as Grade I, which could have positively impacted median ST, since Grade I meningiomas are typically considered "benign" (Sturges et al., 2008). Ideally, future larger studies would investigate a potential relationship between meningioma subtype/grade and ST, which might add useful information for prognostic purposes.

The recurrence rate of intracranial meningiomas in humans is $9-12 \%$ with grossly complete excision and may be as high as $40 \%$ with incomplete excision (Spagnuolo et al., 2003) Meningioma recurrence depends on extension of resection, location and histological grade of the tumor (Schiffer et al., 2005). In our study, the main cause of death was the progression of neurological signs, behavioral changes, and uncontrolled seizures, allegedly related to tumor regrowth, scar tissue formation or brain herniation trough craniectomy defect. In most cases a follow-up MRI to confirm tumor recurrence could not be performed, but the clinical signs and the neurological exam suggested recurrence. Hence, euthanasia due to suspected progression of clinical signs was the most common cause of death in our patients.

Fewer studies document ST for canine gliomas. In humans, gliomas are categorized as Grade I, II, III, and IV according to their histopathologic features and the 2007 WHO classification system, with Grade IV gliomas being the most aggressive type (Louis et al., 2007).

Grade I include: subependymal giant cell astrocytoma and pilocytic astrocytoma. Grade II include: pilomyxoid astrocytoma, diffuse astrocytoma, pleomorphic xanthoastrocytoma, oligodendroglioma and oligoastrocytoma. Grade III include: anaplastic astrocytoma, anaplastic oligodendroglioma and anaplastic oligoastrocytoma. Grade IV, the most aggressive type, include: glioblastoma, giant cell glioblastoma and gliosarcoma (Louis et al., 2007).

To the authors' knowledge, this is the first study reporting ST in canine gliomas treated with SRA showing a median ST of 66 days (mean 117 days). Interestingly, one dog with an anaplastic glioma survived for 2 years after surgery before dying of uncontrolled seizures.

In human medicine, it has been suggested that repeated surgery with maximal extend of resection should be standard of care for recurrent low-grade gliomas (Uppstrom et al., 2016).

In the present study, ST for gliomas appeared slightly longer than the ST that had been previously reported following palliative care (Adams et al., 2005), but considerably shorter than the ST previously reported following radiotherapy or following surgery and radiotherapy (Adams et al., 2005). Furthermore, this type of surgery allows the possibility of a histopathological confirmation and the use of alternatives or adjunctive future therapies, such as radiation therapy or chemotherapy.

There are important factors to consider when comparing the results of ST for meningiomas and gliomas. The different nature and growth patterns of both types of tumors (extra- versus intra-axial) is the most likely factor influencing the differences in ST observed after SRA. Gliomas, being intra-axial tumors, may be more difficult to access because they often have deeper locations than meningiomas; moreover, gliomas may also be more challenging to resect because they typically lack a clear plane of dissection (Niebauer et al., 1991; Rossmeisl, 2014). Another important factor to consider is that most meningiomas included in this study were Grade I, whereas most gliomas were Grade III. Therefore, it is likely that in the present study meningiomas showed a less aggressive biological behavior than gliomas.

Regarding the MRI secondary changes evaluated in this study (midline shift, ventricular compression and peritumoral edema), there was a correlation between the presence of a midline shift and ventricular compression and ST for meningiomas. A study investigated the MRI characteristics of histological confirmed meningiomas and concluded that it was not possible to predict the meningioma subtype or grade based on MRI features, emphasizing the need for histopathology for an antemortem diagnostic confirmation (Sturges et al., 2008). Another study evaluated potential prognostic factors associated with ST in brain tumors and found no significant correlation between the edema observed on MRI and ST, (Rossmeisl et al., 2013) which was also observed in the present study.

At our institution, radiation therapy is always recommended following surgical resection of intracranial tumors; however, radiotherapy was not available in our country during the study period, and owners elected to not pursue this treatment modality due to the logistic difficulties and the expenses that would be associated with pursuing radiotherapy in a different country.

There were several limitations and strengths to our study. Limitations included the retrospective nature of the study, along with a limited sample size. As in all retrospective studies, multiple variables existed that could not be controlled, which might have influenced our results. At the time of the surgery, information regarding whether the excision was considered to be complete or incomplete was not included in the medical records. Thus, it was not possible to correlate if the completeness or not of the surgical resection might have had an influence on recurrence or ST. Euthanasia due to recurrence or progression of clinical signs was the most common cause of death. In most of these 
cases, tumor recurrence was suspected, but a repeat MRI or a postmortem histopathological examination were not performed, which is another limitation of the study.

Our decision to exclude the patients that did not survive pass $>7$ days after surgery was intended to eliminate the direct influence of postsurgical complications on the $\mathrm{ST}$, as well as to be able to compare the results with previously published studies that calculated ST following the same approach (Axlund et al., 2002).

One of the strengths of this study is that all biopsies were obtained during the initial surgical resection. Other previous clinical studies, in particular during the treatment of gliomas, rely on suspected gliomas by MRI characteristics or biopsy or postmortem samples obtained following radiation or chemotherapy, which might change the nature or histopathological grade of those tumors (Brearley et al., 1999; Spugnini et al., 2000; Adams et al., 2005).

Moreover, radiation therapy itself has been reported to induce the formation of brain tumors in people ( Salvati et al., 2003; Umansky et al., 2008; Ecemis et al., 2013) and dogs (Luján Feliu-Pascual et al., 2009).

Our study also provides ST data after SRA for a group of $14 \mathrm{dogs}$ with gliomas. There is very limited information available on ST after SRA for histopathologically confirmed canine gliomas. As such, the results from the present study add extra data to the scant information currently available, and provide additional evidence that neurologists can use when choosing treatment options for presumptive glial tumors.

The results of this study suggest that SRA might be an appropriate treatment, particularly for meningiomas, when radiation therapy is not readily available. Also, the presence of midline shift and ventricular compression might be negative prognostic factors for dogs with meningiomas.

For canine gliomas, this is the first clinical study reporting ST using SRA. Results shows longer survival time with surgery compared to previously reported palliative treatment for glioma. Also, no MRI characteristics appeared to be associated with ST in this group of dogs with gliomas.

Studies that investigate the ST associated with the different therapies available to treat brain tumors, such as the one presented here, provide veterinary neurologists with additional evidence to aid in the decision-making process and to estimate the most likely prognosis for each one of these patients.

\section{Acknowledgments}

No third-party funding or support was received in connection with this study or the writing or publication of the manuscript.

\section{Conflict of interest}

The authors declare that there is no conflict of interest.

\section{References}

Adams, V.J., Platt, S.R., Garosi, L.S., Murphy, S. and Abramson, C.J. 2005. Survival analysis of canine gliomas and meningiomas treated with corticosteroids hypofractionated radiotherapy or surgery and radiotherapy. Proceedings of the Symposium BSAVA, April 7 to 10, Birmingham, UK.

Axlund, T.W., McGlasson, M.L. and Smith, A.N. 2002. Surgery alone or in combination with radiation therapy for treatment of intracranial meningiomas in dogs: 31 cases (1989-2002). J. Am. Vet. Med. Assoc. 221, 1597-1600.

Bagley, R.S., Gavin, P.R., Moore, M.P., SIlver, G.M., Harrington, M.L. and Connors, R.L. 1999. Clinical signs associated with brain tumors in dogs: 97 cases (1992-1997). J. Am. Vet. Med. Assoc. 215, 818819.

Beltran, E., Platt, S.R., McConnell, J.F., Dennis, R., Keys, D.A. and De Risio, L. 2014. Prognostic value of early magnetic resonance imaging in dogs after traumatic brain injury: 50 cases. J. Vet. Intern. Med. 28, 1256-1262.

Bilderback, A., Faissler, D., Sato, A.F., Keating, J.H. and Mc Donnell, J.J. 2006. Transfrontal craniectomy, radiation therapy, and/or chemotherapy in the treatment of canine meningiomas. Proceedings of the American College of Veterinary Internal Medicine Symposium, May 31 to June 3, Louisville, USA, pp: 784.

Bley, C.R., Sumova, A., Roos, M. and Kaser-Hotz, B. 2005. Irradiation of brain tumors in dogs with neurologic disease. J. Vet. Intern. Med. 19, 849854.

Brearley, M.J., Jeffery, N.D., Phillips, S.M. and Dennis, R. 1999. Hypofractionated radiation therapy of brain masses in dogs: a retrospective analysis of survival of 83 cases (1991-1996). J. Vet. Intern. Med. 13, 408-412.

Dickinson, P.J. 2014. Advances in diagnostic and treatment modalities for intracranial tumors. J. Vet. Intern. Med. 28, 1165-1185.

Ecemis, G.C., Atmaca, A. and Meydan, D. 2013. Radiation-associated secondary brain tumors after conventional radiotherapy and radiosurgery. Expert. Rev. Neurother. 13, 557-565.

Evans, S.M., Dayrell-Hart, B., Powlis, W., Christy, G. and VanWinkle, T. 1993. Radiation therapy of canine brain masses. J. Vet. Intern. Med. 7, 216219.

Foster, E.S., Carrillo, J.M. and Patnaik, A.K. 1988. Clinical signs of tumors affecting the rostral cerebrum in 43 dogs. J. Vet. Intern. Med. 2, 71-74.

Greco, J.J., Aiken, S.A. and Berg, J.M. 2006. Evaluation of intracranial meningioma resection 
with a surgical aspirator in dogs:17 cases (19962004). J. Am. Vet. Med. Assoc. 229, 394-400.

Griffin, L.R., Nolan, M.W., Selmic, L.E., Randall, E., Custis, J. and LaRue, S. 2014. Stereotactic Radiation Therapy for Treatment of Canine Intracranial Meningiomas. Vet. Comp. Oncol. 14, 158-170.

Jung, D.-I., Kim, H.-J., Park, C., Kim, J.-W., Kang, B.T., Lim, C.-Y., Park, E.H., Sur, J.H., Seo, M.G., Hahm, D.H. and Park, H.M. 2006. Long-term chemotherapy with lomustine of intracranial meningioma occurring in a miniature schnauzer. J. Vet. Med. Sci. 68, 383-386.

Klopp, L.S. and Rao, S. 2009. Endoscopic-assisted intracranial tumor removal in dogs and cats: longterm outcome of 39 cases. J. Vet. Intern. Med. 23, 108-115.

Koestner, A., Bilzer, T., Fatzer, R., Schulman, F.Y. and Van Winkle, T.J. 1999. World Health Organization histological classification of tumors of the nervous system of domestic animals, in: World Health Organization histological classification of tumors of the nervous system of domestic animals. Second. DC, pp: 27-29.

Kostolich, M. and Dulisch, M.L. 1987. A surgical approach to the canine olfactory bulb for meningioma removal. Vet. Surg. 16, 273-277.

Louis, D.N., Ohgaki, H., Wiestler, O.D., Cavenee, W.K., Burger, P.C., Jouvet, A., Scheithauer, B.W. and Kleihues, P. 2007. The 2007 WHO Classification of Tumours of the Central Nervous System. Acta Neuropathol. 114, 97-109.

Luján Feliu-Pascual, A., Dennis, R., Murphy, S., De Risio, L. and Matasiek, K. 2009. Cerebral necrosis following hypofractionated radiotherapy for canine intracranial tumors: a magnetic resonance imaging and pathological study. In: Proceedings of the 22nd Symposium ESVN- ECVN, September 24 to 26, 2009. Bologna, Italy, pp: 43.

Mandara, M.T., Pavone, S., Brunetti, B. and Mandrioli, L. 2009. A comparative study of canine and feline meningioma classification based on the WHO histological classification system in humans. In: Proceedings of the 22nd Symposium ESVNECVN, September 24 to 26, 2009. Bologna, Italy, pp: 41.

Niebauer, G.W. and Dayrell-Hart, B.L. 1991. Evaluation of craniotomy in dogs and cats. J. Am. Vet. Med. Assoc. 198, 89-95.

Rossmeisl, J.H. 2014. New treatment modalities for brain tumors in dogs and cats. Vet. Clin. North Am. Small Anim. Pract. 44, 1013-1038.

Rossmeisl, J.H., Jones, J.C., Zimmerman, K.L. and Robertson, J.L. 2013. Survival time following hospital discharge in dogs with palliatively treated primary brain tumors. J. Am. Vet. Med. Assoc, 242,
193-198.

Salvati, M., Frati, A., Russo, N., Caroli, E., Polli, F.M., Minniti, G. and Delfini, R. 2003. Radiation-induced gliomas: report of 10 cases and review of the literature. Surg. Neurol. 60, 60-67.

Schiffer, D., Ghimenti, C. and Fiano, V. 2005. Absence of histological signs of tumor progression in recurrences of completely resected meningiomas. J. Neurooncol. 73, 125-130.

Sessums, K. and Mariani, C. 2009. Intracranial meningioma in dogs and cats: a comparative review. Compend. Contin. Educ. Vet. 31(7), 330339.

Snyder, J.M., Shofer, F.S., Van Winkle, T.J. and Massicotte, C. 2006. Canine intracranial primary neoplasia: 173 cases (1986-2003). J. Vet. Intern. Med. 20, 669-675.

Song, R.B., Vite, C.H., Bradley, C.W. and Cross, J.R. 2013. Postmortem evaluation of 435 cases of intracranial neoplasia in dogs and relationship of neoplasm with breed, age, and body weight. J. Vet. Intern. Med. 27, 1143-1152.

Spagnuolo, E., Calvo, A., Erman, A. and Tarigo, A. 2003. Recurrent meningiomas with progressive aggressiveness and posterior extracranial extension. Neurocirugia (Astur). 14, 409-416.

Spugnini, E.P., Thrall, D.E., Price, G.S., Sharp, N.J., Munana, K. and Page, R.L. 2000. Primary irradiation of canine intracranial masses. Vet. Radiol. Ultrasound. 41, 377-380.

Sturges, B.K., Dickinson, P.J., Bollen, A.W., Koblik, P.D., Kass, P.H., Kortz, G.D., Vernau, K.M., Knipe, M.F., Lecouteur, R.A. and Higgins, R.J. 2008. Magnetic resonance imaging and histological classification of intracranial meningiomas in 112 dogs. J. Vet. Intern. Med. 22, 586-595.

Tamura, S., Tamura, Y., Ohoka, A., Hasegawa, T. and Uchida, K. 2007. A canine case of skull base meningioma treated with hydroxyurea. J. Vet. Med. Sci. 69, 1313-1315.

Turrel, J.M., Fike, J.R., LeCouteur, R.A., Pflugfelder, C.M. and Borcich, J.K. 1984. Radiotherapy of brain tumors in dogs. J. Am. Vet. Med. Assoc. 184, 8286.

Umansky, F., Shoshan, Y., Rosenthal, G. and Fraifeld, S. 2008. Radiation-induced meningioma, Neurosurg. Focus. 24, E7.

Uppstrom, T.J., Singh, R., Hadjigeorgiou, G.F., Magge, R. and Ramakrishna, R. 2016. Repeat surgery for recurrent low-grade gliomas should be standard of care. Clin. Neurol. Neurosurg. 151, 18-23.

Van Meervenne, S. and Verhoeven, P.S. 2014. Comparison between symptomatic treatment and lomustine supplementation in $71 \mathrm{dogs}$ with intracranial, space-occupying lesions. Vet. Comp. Oncol. 12, 66-77. 